\title{
Concurrent Paraneoplastic Dermatomyositis and Acquired C1 Esterase Inhibitor Deficiency in Primary Laryngeal Small Cell Carcinoma
}

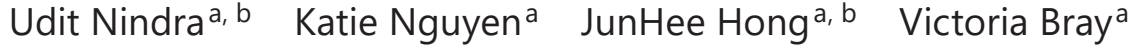 \\ Eugene Moylan ${ }^{a}$ \\ aMedical Oncology Department, Liverpool Hospital, Liverpool, NSW, Australia; 'bSchool of \\ Medicine, University of New South Wales, Kensington, NSW, Australia
}

\section{Keywords}

Dermatomyositis · Head and neck cancer · Immunology · Small cell carcinoma

\begin{abstract}
Small cell carcinoma is associated with a number of paraneoplastic syndromes. We report a case of a 42-year-old female who presented with primary laryngeal small cell carcinoma associated with concurrent paraneoplastic dermatomyositis and paraneoplastic angioedema secondary to acquired $\mathrm{C} 1$ esterase inhibitor deficiency. The patient required extensive treatment for her dermatomyositis including high-dose corticosteroid therapy and intravenous immunoglobulin followed by steroid-sparing disease-modifying immunosuppression. Her angioedema also required multiple lines of therapy including bradykinin inhibitors and human recombinant $\mathrm{C} 1$ esterase. We believe this is the first reported case of either of these paraneoplastic syndromes arising from an extrapulmonary small cell carcinoma and highlights the difficulty of its initial diagnosis as well as concurrent management.
\end{abstract}

\section{Introduction}

Extrapulmonary small cell carcinomas account for up to $5 \%$ of all small cell cancer diagnosis [1]. Laryngeal small cell carcinoma is a rarer entity, accounting for $<0.5 \%$ of all primary laryngeal cancer diagnoses [2]. Paraneoplastic syndromes are known complications of small 
cell malignancies, occurring in $1-7.4 \%$ of cases [3]. The majority of the syndromes are endocrine related with rheumatological and immunological complications known to be less frequently encountered.We are reporting a case of a young 43-year-old woman who presented with primary laryngeal small cell carcinoma complicated by both paraneoplastic dermatomyositis (DM) and paraneoplastic-acquired C1 esterase inhibitor deficiency, who had a protracted hospital admission requiring immunomodulatory treatments. We believe this is the first published case of either paraneoplastic DM or paraneoplastic-acquired C1 esterase inhibitor deficiency originating from a laryngeal origin small cell carcinoma.

\section{Initial Presentation and Diagnosis}

A 43-year-old woman was admitted to our hospital following a 2-month history of right lateral neck swelling that was being investigated by her family physician. She described her swelling as progressive, impacting her breathing with orthopnoea, causing significant dysphagia with odynophagia and concurrent hoarseness of her voice. She did not have any systemic features at the time of presentation such as fevers, abdominal pain, night sweats, alteration in bowel habits, nausea, vomiting, or cough. The patient did not have any other significant past medical history, was not on any medications, and had no allergies to note. She was a functionally independent non-smoker with no history of alcohol or drug use. Prior to hospital presentation, she had undergone an outpatient ultrasound which showed evidence of widespread lymphadenopathy in the right cervical region. Given her initial degree of facial swelling, she was commenced on dexamethasone and underwent a CT of her neck to rule out superior vena cava obstruction (SVCO). Although her CT ruled out SVCO, it did show evidence of significant lymphadenopathy in the right cervical chain and abnormal growth around the laryngeal wall. Given concerns about malignancy, she also underwent systemic CT scans which showed diffuse disease uptake in the liver in addition to her larynx (Fig. 1).

She underwent a nasoendoscopy and biopsy of her right false vocal cord as well as a biopsy of her liver. Histopathology from both lesions showed a poorly differentiated carcinoma consisting of cells with scanty cytoplasm, hyperchromatic nuclei, and nuclear moulding. The immunohistochemistry was positive for AE1/3, MNF116, and CD56 whilst negative for TTF1,

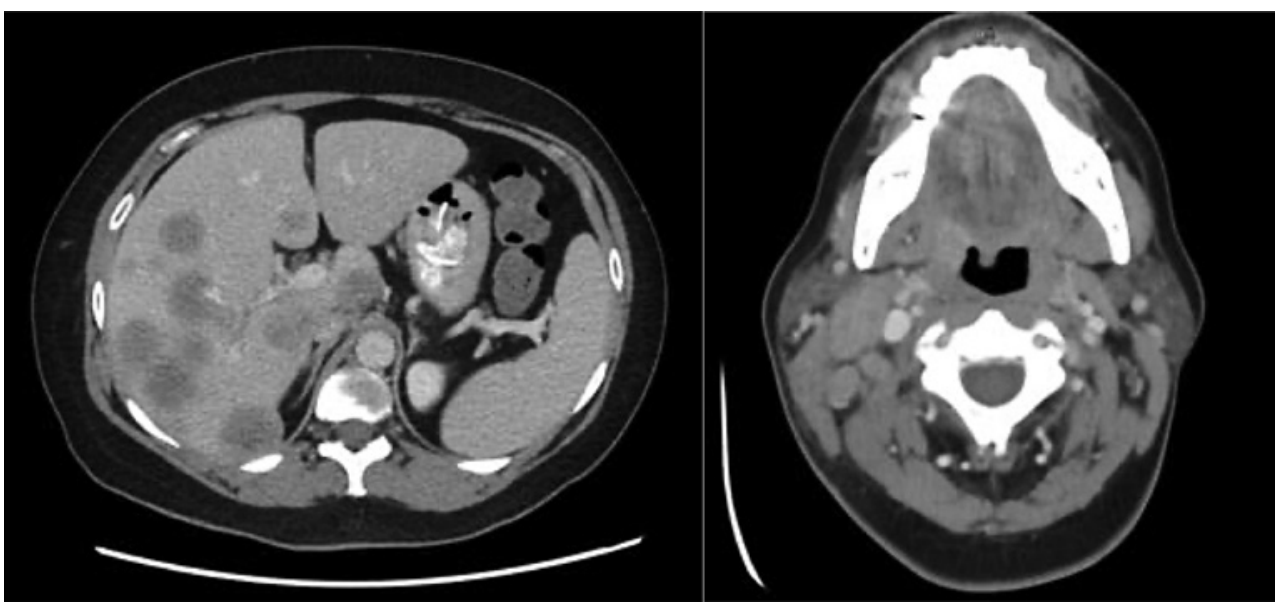

Fig. 1. Diffuse scattered lymphadenopathy can be seen in the right cervical chain with evidence of distant metastases seen in the liver. 
Fig. 2. Liver biopsy consistent with small cell neuroendocrine tumour with stain showing positivity for CD56 and CK7, negative for TTF1, and a Ki67 of 75\%.

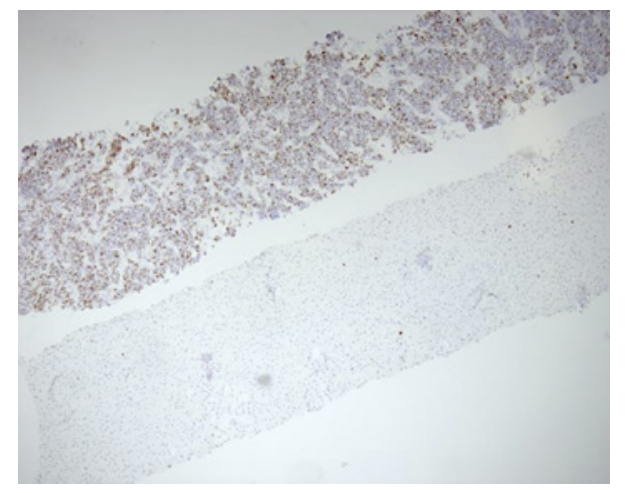

synaptophysin, p40, CK14, and p16. The Ki67 was 75\%. Overall, the appearances were consistent with a small cell neuroendocrine carcinoma (Fig. 2).

Following her biopsy, she underwent systemic imaging which included a computed tomography (PET-CT) scan of her chest, abdomen, and pelvis that revealed evidence of diffuse cervical nodal uptake as well as intensely avid lesions scattered throughout the liver (Fig. 3a, b). There was no evidence of any disease within the lung. She underwent further biopsies of the liver lesions which confirmed the same histopathological phenotype as the laryngeal lesions. She was subsequently diagnosed with extensive-stage small cell cancer originating from her larynx.

At the time of initial presentation, the patient had complained of generalized myalgia without obvious weakness, and this was initially felt to be in keeping with her systemic disease. She also had marked facial swelling, and given her CT did not show any evidence of SCVO, this was felt to be possibly due to lymphatic obstruction. She underwent her first chemotherapy (cisplatin $80 \mathrm{mg} / \mathrm{m}^{2}$ and etoposide $100 \mathrm{mg} / \mathrm{m}^{2}$ ) without any specific complications encountered. On cycle 1 day 4 , she was discharged home only for her to present back to the emergency department $48 \mathrm{~h}$ later with ongoing progressive facial swelling as well as significant systemic weakness, myalgia, and malaise. Her neck region was re-imaged at this time to again exclude SVCO, and this was again noted to be negative. Her examination at this time showed generalized tenderness on muscle bulk palpation, no obvious rashes on the face or scalp, and no arthritic pain but evidence of $4 / 5$ weakness in her flexion and extension of the wrist and fingers as well as 4/5 weakness in knee flexion, knee extension, ankle flexion, and ankle dorsiflexion.

Given these findings were not in keeping with either a chemotherapy or anatomical cancer effect, a possible paraneoplastic phenomenon was suspected. Her creatinine kinase level was found to be markedly elevated at 4,100 U/L. After consultation with both immunology and rheumatology, she was suspected of having paraneoplastic DM and was commenced on a 3-day course of $750 \mathrm{mg}$ intravenous methylprednisolone followed by high-dose prednisone at $80 \mathrm{mg}$ daily with proton pump inhibitor cover. Her DM panel revealed a strongly positive TIF1-gamma antibody and positive SAE-1 antibody, consistent with her disease. Her ANA was positive $(>1: 1,280)$ with a DFS-70 pattern on her nuclear antibodies. Her extractable nuclear antigen panel was negative.

Furthermore, her degree of facial swelling was also felt to be unusual from either the cancer or the DM, and a suspected diagnosis of acquired angioedema secondary to paraneoplastic C1 esterase inhibitor deficiency (C1INH-AAE) was made. She was initially treated with icatibant $30 \mathrm{mg}$ daily for 3 days. However, due to a lack of significant improvement, she was then trialled on intravenous immunoglobulin with Intragram 10\%, $80 \mathrm{mg}$, over 2 days. Despite a further lack of response, she was then given Berinert, a plasma-derived concentrate of 


\section{Case Reports in Oncology}

\begin{tabular}{l|l}
\hline Case Rep Oncol 2021;14:1806-1813 \\
\hline DOI: 10.1159/000520383 & $\begin{array}{l}\text { @ 2021 The Author(s). Published by S. Karger AG, Basel } \\
\text { www.karger.com/cro }\end{array}$ \\
\hline
\end{tabular}

Nindra et al.: Concurrent Paraneoplastic Dermatomyositis in Laryngeal Small Cell Carcinoma
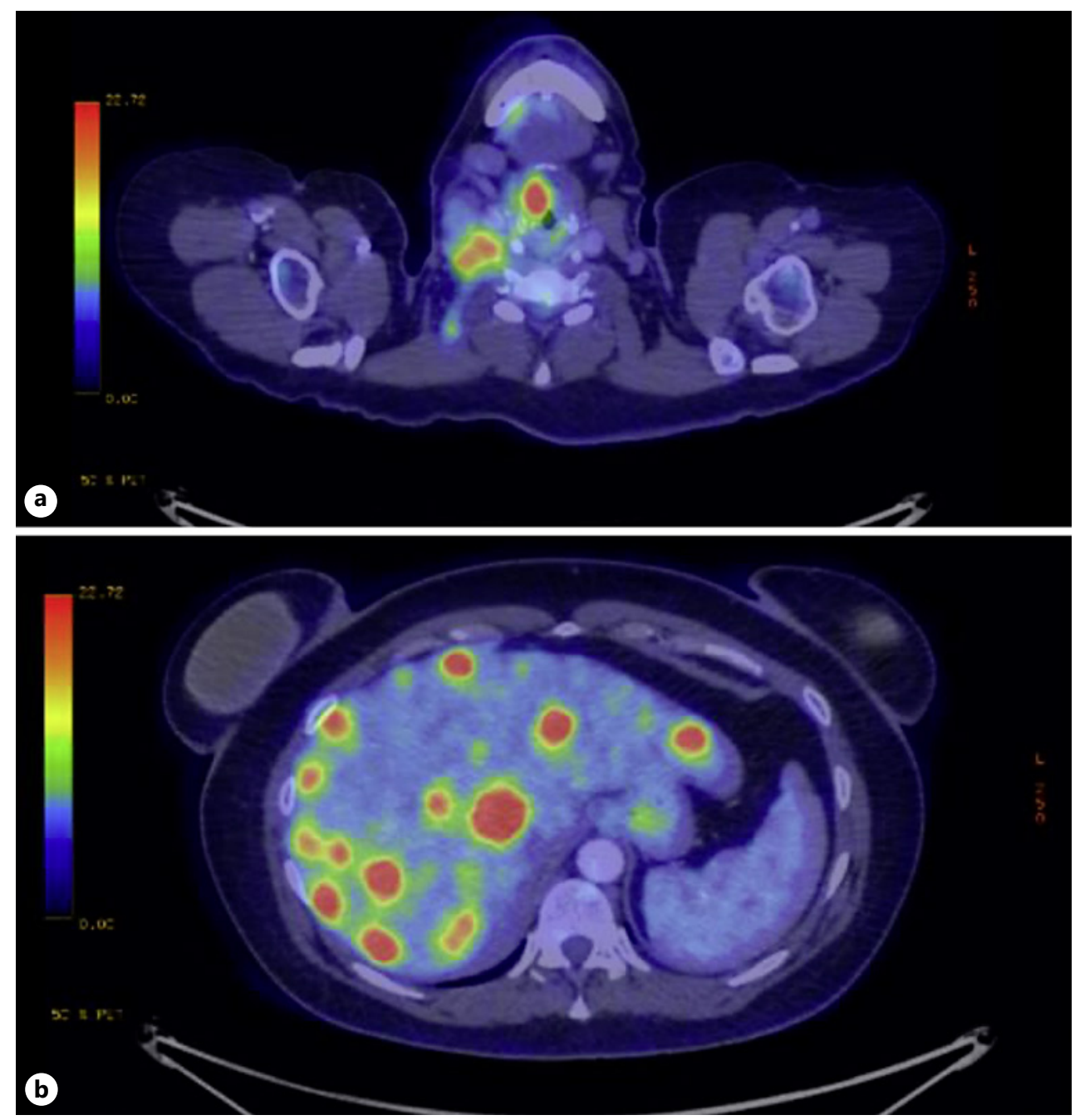

Fig. 3. a Intensely FDG avid oval soft tissue mass lesion within the right side of the larynx extended to the supraglottic region measuring $12 \times 1 \times 7 \times 18 \mathrm{~mm}$ which is intensely FDG avid (SUV 12.2). b There are innumerable intensely FDG avid intrahepatic metastatic deposits measuring up to 20 and $23 \mathrm{~mm}$ in diameter in segment 6 (SUV 12.5 and 10.0) and the left lobe of the liver in segment 3, $21 \mathrm{~mm}$ (SUV 13.8).

human C1 esterase inhibitor, which markedly improved her facial oedema and also confirmed the diagnosis of paraneoplastic $\mathrm{C} 1$ esterase inhibitor deficiency. Her laboratory analysis was not available until after this treatment had been initiated but interestingly did not show evidence of low C1 esterase inhibitor $(0.40 \mathrm{~g} / \mathrm{L}$, range $0.18-0.32 \mathrm{~g} / \mathrm{L})$ nor any functional impairment of $\mathrm{C} 1$ esterase. Furthermore, her $\mathrm{C} 1$ esterase antibodies were also found to be low $(115 \mu / \mathrm{L}$, range $118-244 \mu / \mathrm{L})$. The remainder of her panel was normal including IgA, IgG, IgM, C3, and C4 levels.

Her admission was further complicated by development of autoimmune haemolytic anaemia with directantigen positivity noted after a significant drop in haemoglobin. Following specialist haematology input, this was suspected to be likely secondary to her IVIG therapy. Fortunately, she did not require any active treatment for this, and it self-resolved. The patient underwent a muscle biopsy of her right lateral thigh 14 days after initiation of immunosuppressive therapy which showed strong and diffuse MHC-1 upregulation which was consistent with an autoimmune myopathy. However, there was notably minimal necrosis and regeneration 


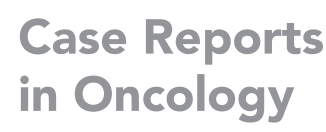

\begin{tabular}{l|l}
\hline Case Rep Oncol 2021;14:1806-1813 \\
\hline DOI: 10.1159/000520383 & $\begin{array}{l}\text { C) 2021 The Author(s). Published by S. Karger AG, Basel } \\
\text { www.karger.com/cro }\end{array}$ \\
\hline
\end{tabular}

Nindra et al.: Concurrent Paraneoplastic Dermatomyositis in Laryngeal Small Cell Carcinoma

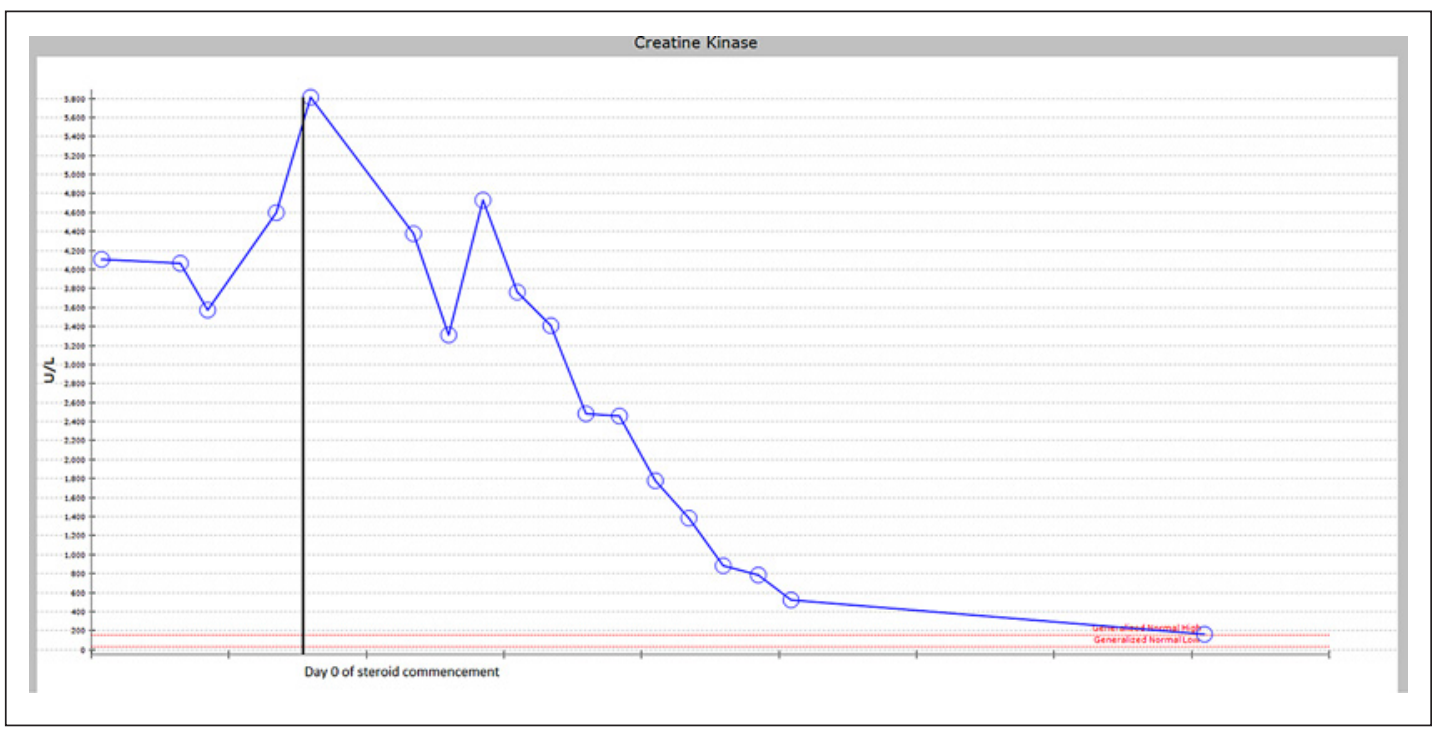

Fig. 4. Creatinine kinase trend over patient's hospital admission. Steroid therapy was commenced on April 26 with methylprednisolone $750 \mathrm{mg}$ daily for 3 days.

to account for the degree of elevated creatinine kinase. She continued on steroids with the aim to slowly wean her course by $10 \mathrm{mg}$ reduction every 2 weeks. During this time, her creatinine kinase was shown to significantly improve (Fig. 4).

Given concerns about the ongoing use of steroids, multidisciplinary discussions were had between oncology, immunology, and rheumatology with regard to her DM management, and a decision to commence methotrexate as a steroid-sparing disease-modifying agent was made. Given the length of her hospital admission, the patient was able to complete cycle 2 of her cisplatin/etoposide during this inpatient admission. She was discharged 4 weeks following her presentation and is currently undergoing further chemotherapy for her laryngeal small cell carcinoma. She has not required further up-titration of her steroid dosages, and her facial swelling has improved.

\section{Discussion}

\section{Paraneoplastic Dermatomyositis}

Paraneoplastic syndromes arise from tumour secretion of bioactive hormones, peptides, or cytokines that result in a myriad of syndromes across multiple body systems. These can range from neurological, endocrinological, dermatological, rheumatological, and haematological manifestations. Most commonly associated malignancies include small cell carcinomas of the lung and breast, gynaecological tumours, and haematological malignancies [4-6].

Of these known syndromes, DM is a rare inflammatory condition that characteristically presents with a pathognomonic violaceous eruption over the upper eyelids (often described as a heliotrope rash) and Gottron papules (a scaly rash over bony prominences that often appears psoriatic). Other cutaneous manifestations include the poikiloderma over extensor surfaces of the neck and back (known as a positive "shawl" sign), periungual telangectasia, facial swelling (i.e., often confused for angioedema), and diffuse erythematous alopecia [4, 5, 7]. Patients characteristically complain of symmetrical and progressive proximal myopathy which is reflected in a notable rise in serum creatinine kinase $[7,8]$.

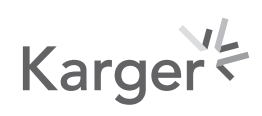


Nindra et al.: Concurrent Paraneoplastic Dermatomyositis in Laryngeal Small Cell Carcinoma

DM is known to be associated with underlying malignancy, with roughly $20-25 \%$ of cases of DM associated with an oncological diagnosis. Sigurgeirsson et al. [9] have calculated a relative risk of 2.4 in males with DM and 3.4 for females. Associated primary sites of association have been identified as ovarian and breast primary tumours in women and prostate and lung adenocarcinoma in men [4-6]. Diagnosis of DM is typically a combination of clinical cutaneous signs, proximal myopathy, and exclusion of other possible dermatological conditions through skin biopsy $[4,8]$. Serological investigations that support a diagnosis of DM include elevated creatinine kinase or lactate dehydrogenase level, as well as positive anti-pp115/140 (anti-TIF1) IgG antibodies support a diagnosis of paraneoplastic DM [10]. Diagnostic confirmation with electromyology, muscle MRI, and/or muscle biopsy can be utilized if the diagnosis is not clear [8].

The main treatment principle of malignancy-related DM is effective cancer treatment and concomitant immunosuppressive therapy. Successful tumour-related therapy will ameliorate symptoms; however, up to one-third of patients with DM will experience significant residual motor impairment [4]. Generally, first-line immunosuppressive therapy commences at a starting dose of prednisolone $0.5-1 \mathrm{mg} / \mathrm{kg}$ daily. A trial of pulse methylprednisolone can be trialled in severe cases. Creatinine kinase and lactic dehydrogenase levels often correlate with response to therapy. Often other steroid-sparing immunosuppressive agents (options including methotrexate, azathioprine, cyclophosphamide, or mycophenolate) can be trialled to prevent significant long-term steroid-related effects. Unfortunately, therapy for cutaneous manifestations of DM often persists despite immunosuppressive therapy, and topical therapy has limited efficacy $[7,8]$.

Our patient presented with cutaneous eruptions and complaints of myalgia that were nonspecific. Although our patient demonstrated a heliotrope rash of the upper eyelids, this was associated with generalized facial oedema, which was initially thought to be a manifestation of acquired angioedema secondary to C1 esterase deficiency (discussed below). On review of the patient's ongoing complaints of pain localized to the anterior chest and back, the patient demonstrated characteristic shawl sign and Gottron papules accompanied by progressive muscle weakness, prompting further investigation. The raised creatinine kinase level, positive TIF1gamma antibody, and positive SAE-1 antibody supported a diagnosis of paraneoplastic DM.

In consultation with the immunology team, the decision was made to commence a 3-day course of high-dose intravenous methylprednisolone and high-dose prednisone therapy to good effect, as reflected in the steep decrease in serum creatinine kinase level in Figure 4. In keeping with the natural history of paraneoplastic DM, whilst our patient's myositis improved significantly, her cutaneous symptoms required prolonged topical steroid therapy and local analgesia prior to slow improvement.

Published case reports of paraneoplastic syndromes associated with laryngeal cancers are rare, all arising from supraglottic squamous cell carcinomas $[11,12]$. This is the first that is published that originates from a small cell carcinoma of laryngeal primary.

\section{Paraneoplastic-Acquired Angioedema Secondary to C1 Esterase Deficiency}

C1INH-AAE is characterized by angioedema which often affects the face, upper respiratory tract, legs, hands, and gastrointestinal tract. It most commonly arises after the 2 nd decade of life and is often associated with underlying autoimmune conditions [13]. It is caused by the unchecked production of bradykinin, a vasodilator, which leads to smooth muscle relaxation in the walls of blood vessels with resultant surrounding oedema [14].

Although different variations of the condition exist, the diagnosis remains a combination of clinical features with supportive laboratory evidence with either deficiency in C1INH or alteration of C1INH function [15]. Interestingly, in our patient, there was neither a deficiency in C1INH identified nor evidence of malfunctional protein status. Furthermore, typically in C1INH-AAE, C1q levels are also shown to be lowered, but in our patient, the levels were normal

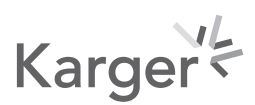



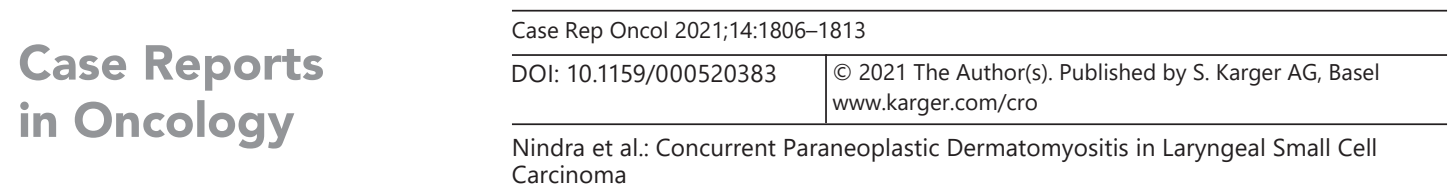

(135 $\mu / \mathrm{L}$, range 118-244 $\mu / \mathrm{L})$. However, despite these laboratory findings, her clinical syndrome and treatment response undoubtedly confirmed her pathological angioedema.

AAE that is associated with $\mathrm{C} 1 \mathrm{INH}$ is rare, noted to be $>10$ times rarer than hereditary forms of the disease which are estimated to occur in between 1/10,000 and 1/50,000 of the population [16]. However, C1INH-AAE in the setting of malignancy is not a new phenomenon. In a retrospective nationwide study done in France where 92 cases of acquired disease were reviewed, it was found that 44 cases were due to non-Hodgkin lymphoma, 3 due to multiple myeloma, and 1 due to bronchial adenocarcinoma [17]. However, from our literature search, there have been no previously documented cases of C1INH-AAE as a secondary paraneoplastic phenomenon due to small cell carcinoma.

Multiple treatment modalities are available for C1INH-AAE. The first is icatibant, an antagonist of the bradykinin B2 receptor which was the initial treatment of choice in our patient given the acuity of her laryngeal oedema. Multiple trials including the FAST-3 has shown evidence that icatibant significantly reduces median response times for reduction in symptom severity compared with placebo (1.5 vs. $18.5 \mathrm{~h}$ ) [18]. In previous trials, icatibant has been shown to be highly effective, producing responses in $>95 \%$ of cases [17]. However, given our patient's lack of response, she was subsequently treated with the C1 inhibitor concentrate, Berinert. This is the most widely used therapy for C1INH-AAE, effective in $>90 \%$ of patients $[17,19]$. One consideration to keep in mind for patients is that over the long term, there is risk of resistance to therapy which is suspected to be due to rapid destruction of the inhibitor protein, although no data are formally available to confirm this.

\section{Conclusion}

Laryngeal small cell carcinoma is a rare entity. Our case highlights rare paraneoplastic complications of small cell carcinoma occurring simultaneously together which made both diagnosis and management challenging. Further studies into the epidemiology of paraneoplastic immunological and rheumatological conditions would be helpful to improve clinician awareness.

\section{Acknowledgment}

In addition to the authors listed above, we would like to acknowledge the Liverpool Hospital Anatomical Pathology Department for providing figures used in this text.

\section{Statement of Ethics}

Formal ethical approval was not required for case report composition requirement as per the South West Sydney Human Resources and Ethics Committee. Written and verbal consent was the mandatory requirement. Given the patient was deceased at the time of publication written and verbal informed consent was obtained from participant's next-of-kin for publication of the details of their medical case and any accompanying images.

\section{Conflict of Interest Statement}

The authors have no conflicts of interest to declare.

\section{Karger'₹}




\section{Case Reports in Oncology}

\begin{tabular}{l|l}
\hline Case Rep Oncol 2021;14:1806-1813 \\
\hline DOI: 10.1159/000520383 & $\begin{array}{l}\text { ○ 2021 The Author(s). Published by S. Karger AG, Basel } \\
\text { www.karger.com/cro }\end{array}$ \\
\hline
\end{tabular}

Nindra et al.: Concurrent Paraneoplastic Dermatomyositis in Laryngeal Small Cell Carcinoma

\section{Funding Sources}

Nil funding was required or sought for the project.

\section{Author Contributions}

All authors were involved in the original report composition and editing aspects of the article. Victoria Bray and Eugene Moylan were additionally responsible for supervision of the project as the senior most members of the research team.

\section{Data Availability Statement}

All data generated or analysed during this study are included in this article. Further enquiries can be directed to the corresponding author.

\section{References}

1 van der Heijden HF, Heijdra YF. Extrapulmonary small cell carcinoma. South Med J. 2005;98:345.

2 Ferlito A. Diagnosis and treatment of small cell carcinoma of the larynx: a critical review. Ann Otol Rhinol Laryngol. 1986;95(6):590-600.

3 Soomro Z, Youssef M, Yust-Katz S, Jalali A, Patel AJ, Mandel J. Paraneoplastic syndromes in small cell lung cancer. J Thorac Dis. 2020;12(10):6253-63.

4 Pelosof LC, Gerber DE. Paraneoplastic syndromes: an approach to diagnosis and treatment. Mayo Clin Proc. 2010;85(9):838-54.

5 Zerdes I, Tolia M, Nikolaou M, Tsoukalas N, Velentza L, Hajiioannou J, et al. How can we effectively address the paraneoplastic dermatomyositis: diagnosis, risk factors and treatment options. J buon. 2017;22(4):1073-80.

6 Hill CL, Zhang Y, Sigurgeirsson B, Pukkala E, Mellemkjaer L, Airio A, et al. Frequency of specific cancer types in dermatomyositis and polymyositis: a population-based study. Lancet. 2001;357(9250):96-100.

7 András C, Ponyi A, Constantin T, Csiki Z, Szekanecz E, Szodoray P, et al. Dermatomyositis and polymyositis associated with malignancy: a 21-year retrospective study. J Rheumatol. 2008;35(3):438-44.

8 Callen JP, Wortmann RL. Dermatomyositis. Clinic Dermatol. 2006;24(5):363-73.

9 Sigurgeirsson B, Lindelöf B, Edhag 0, Allander E. Risk of cancer in patients with dermatomyositis or polymyositis. A population-based study. N Engl J Med. 1992;326(6):363-7.

10 Lu X, Yang H, Shu X, Chen F, Zhang Y, Zhang S, et al. Factors predicting malignancy in patients with polymyositis and dermatomyostis: a systematic review and meta-analysis. PLoS One. 2014;9(4):e94128.

11 Rinaldo A, Coca-Pelaz A, Silver CE, Ferlito A. Paraneoplastic syndromes associated with laryngeal cancer. Adv Ther. 2020;37(1):140-54.

12 Kusunoki T, Homma H, Kidokoro Y, Yanai A, Ikeda K, Wada R. A case of laryngeal cancer associated with dermatomyositis. Clin Pract. 2015;5(4):818.

13 Sinclair D, Smith A, Cranfield T, Lock RJ. Acquired C1 esterase inhibitor deficiency or serendipity? The chance finding of a paraprotein after an apparently low C1 esterase inhibitor concentration. J Clin Pathol. 2004;57(4): 445.

14 Kothari ST, Shah AM, Botu D, Spira R, Greenblatt R, Depasquale J. Isolated angioedema of the bowel due to C1 esterase inhibitor deficiency: a case report and review of literature. J Med Case Rep. 2011;5:467.

15 Agostoni A, Aygören-Pürsün E, Binkley KE, Blanch A, Bork K, Bouillet L, et al. Hereditary and acquired angioedema: problems and progress: proceedings of the third C1 esterase inhibitor deficiency workshop and beyond. J Allergy Clin Immunol. 2004;114(3):S51-131.

16 Longhurst H, Cicardi M. Hereditary angio-oedema. Lancet. 2012;379(9814):474-81.

17 Gobert D, Paule R, Ponard D, Levy P, Frémeaux-Bacchi V, Bouillet L, et al. A nationwide study of acquired C1-inhibitor deficiency in France: characteristics and treatment responses in 92 patients. Medicine. 2016; 95(33):e4363.

18 Lumry WR, Li HH, Levy RJ, Potter PC, Farkas H, Moldovan D, et al. Randomized placebo-controlled trial of the bradykinin $B_{2}$ receptor antagonist icatibant for the treatment of acute attacks of hereditary angioedema: the FAST-3 trial. Ann Allergy Asthma Immunol. 2011;107(6):529-37.

19 Bork K, Staubach-Renz P, Hardt J. Angioedema due to acquired C1-inhibitor deficiency: spectrum and treatment with C1-inhibitor concentrate. Orphanet J Rare Dis. 2019;14(1):65. 